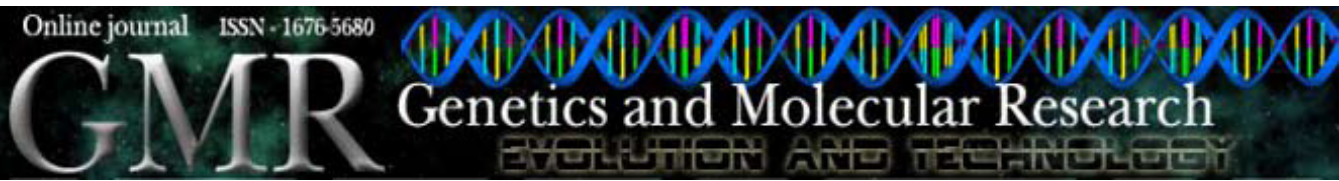

\title{
Analysis and comparison of clinicohematological parameters and molecular and cytogenetic response of two $\mathrm{Bcr} / \mathrm{Abl}$ fusion transcripts
}

\author{
S. Polampalli ${ }^{1}$, A. Choughule ${ }^{2}$, N. Negi ${ }^{1}$, S. Shinde ${ }^{2}$, C. Baisane ${ }^{3}$, P. Amre , $^{3}$ \\ P.G. Subramanian ${ }^{2}$, S. Gujral ${ }^{2}$, K. Prabhash ${ }^{1}$ and P. Parikh ${ }^{1}$ \\ ${ }^{1}$ Molecular Biology Laboratory, Department of Medical Oncology, \\ Tata Memorial Hospital, Mumbai, India \\ ${ }^{2}$ Molecular Biology Laboratory, Department of Hematopathology, \\ Tata Memorial Hospital, Mumbai, India \\ ${ }^{3}$ Cancer Cytogenetics Laboratory, Tata Memorial Hospital, Mumbai, India \\ Corresponding author: S. Polampalli \\ E-mail: drsunitap@yahoo.co.in
}

Genet. Mol. Res. 7 (4): 1138-1149 (2008)

Received June 23, 2008

Accepted August 18, 2008

Published October 21, 2008

\begin{abstract}
Different forms of $p 210$ are produced by alternative splicing, namely $b 2 a 2$ and $b 3 a 2$. There have been many contrasting data establishing a relationship between the two $\mathrm{Bcr} / \mathrm{Abl}$ transcripts and platelet counts and also response to treatment. However, the data published to date have been on a small group of patients. The aim of the present study was to determine whether there was any difference between clinical and hematological parameters at diagnosis between the two $B c r / A b l$ fusion transcripts in our population, and whether the two transcripts responded differently or similarly to imatinib treatment. RT-PCR was performed in 202 cases for detection of $\mathrm{Bcr} / \mathrm{Abl}$ transcripts in newly diagnosed chronic myelogenous leukemia cases in one year. The two transcripts were compared and correlated with clinical, hematological and FISH data and with response to treatment. A total of 138 cases were of $b 3 a 2$ and 64 were of b2a2 transcript. There was no correlation between the hemato-
\end{abstract}


logical parameters and the type of transcript. There was a significant association of blast crisis with $b 2 a 2$, especially with myeloid blast crisis. When compared to FISH results, $10 \%$ of $b 3 a 2$ were found to have a significant association with ${ }^{\prime} \mathrm{Abl}$ deletion as compared to $3 \%$ of $b 2 a 2$. On analyzing the therapeutic response, we did not find any difference between the two transcripts. In conclusion, our findings confirm that the $b 3 a 2$ type transcript is not significantly associated with thrombocytosis, that the short transcript, $b 2 a 2$, occurs with acute phase, i.e., blast crisis, and that there is no difference in treatment response between the two transcripts. However, further studies are required to understand the molecular pathways involved in the $B c r / A b l$ mechanism.

Key words: $B c r / A b l$ fusion transcripts; $b 3 a 2 ; b 2 a 2$; Thrombocytosis; Molecular response

\section{INTRODUCTION}

The $\mathrm{Ph}$ chromosome is a shortened chromosome 22 resulting from a reciprocal translocation, $\mathrm{t}(9 ; 22)(\mathrm{q} 34 ; \mathrm{q} 11)$, between the long arms of chromosomes 9 and 22 (Nowell and Hungerford, 1960; Rowley, 1973). It is the hallmark of chronic myelogenous leukemia $(\mathrm{CML})$ and is found in up to $95 \%$ of patients. It is also found in 5\% of children and in 15 to $30 \%$ of adults with acute lymphoid leukemia and in $2 \%$ of patients with newly diagnosed acute myeloblastic leukemia (Kurzrock et al., 1988; Specchia et al., 1995). The Ph translocation adds a 3' segment of the $\mathrm{Abl}$ gene from chromosome 9q34 to the 5' part of the $\mathrm{Bcr}$ gene on chromosome 22q11, creating a hybrid $B c r / A b l$ gene that is transcribed into a chimeric $B c r / A b l$ messenger RNA (mRNA), which is translated into a chimeric protein of $210 \mathrm{kDa}$ called $p 210 \mathrm{Bcr} / \mathrm{Abl}$. The $\mathrm{Abl}$ gene encodes a non-receptor tyrosine kinase with a molecular mass of $145 \mathrm{kDa}(p 145 \mathrm{Abl})$. It has 11 exons and spans over $230 \mathrm{~kb}$. The breakpoint in the $A b l$ gene occurs usually 5' (toward the centromere) of exon 2 of $A b l$. The $A b l$ exons 2 to 11 (also called a2 to a11) are transposed into the major breakpoint cluster region (M-bcr) of the $B c r$ gene on chromosome 22 between exons 12 and 16 (also referred to as b1 to b5), which extends over $5.8 \mathrm{~kb}$ (Kurzrock et al., 1988). The breakpoint locations within $B c r$ fall either 5' between exons b2 and b3 or 3' (toward the telomere) between exons b3 and b4. A $B c r / A b l$ fusion gene with a $b 2 a 2$ or $b 3 a 2$ junction is created and transcribed into an $8.5-\mathrm{kb}$ mRNA. The differences lead to the lack of the 75-bp b3 exon in the latter rearranged $\mathrm{Bcr} /$ $A b l$ transcript. In 5\% of cases, alternative splicing events allow the expression of both fusion products (Melo, 1996b).

There have been many contrasting data establishing a relationship between the two $\mathrm{Bcr} /$ $A b l$ transcripts and platelet counts and also response to treatment. However, the data published to date have been on a small group of patients. In this study, we determined the clinicohematological and molecular parameters in a large population to validate the results published so far.

The objective of the present study was to determine whether there is any difference in clinical and hematological parameters at diagnosis between the two $B c r / A b l$ fusion transcripts in our population, and whether the two transcripts respond differently or similarly to imatinib treatment. 


\section{MATERIAL AND METHODS}

\section{Patient population}

A total of 202 patients were diagnosed with CML by reverse transcriptase-polymerase chain reaction (RT-PCR) in one year. These cases were studied retrospectively for the two isoforms of $B c r / A b l$ fusion transcript, i.e., $b 3 a 2$ and $b 2 a 2$ for their clinicohematological parameters in terms of age, gender, size of liver, and spleen and baseline hemogram, as per availability of data (for the sake of uniformity, the ratio of the two transcripts has been mentioned for all parameters studied). All hematological parameters including hemoglobin level, platelet count, total leukocyte count (TLC), and bone marrow blast, basophil, and eosinophil percentages were analyzed by accepted criteria and defined ranges (Lewis, 2001; Kratz et al., 2004). Of the 202 cases diagnosed by RT-PCR at baseline, 195 cases were compared with fluorescent in situ hybridization (FISH). FISH analyses for the $B c r / A b l$ fusion transcripts were performed by established techniques on bone marrow cells (Acar et al., 1997)

\section{RT-PCR}

The same bone marrow aspirate provided mononucleated cells for RT-PCR analysis. Mononuclear cells were collected from the bone marrow by the RBC lysing method. Total cellular RNA was extracted, and cDNA was synthesized and then subjected to RT-PCR amplification as described elsewhere (Kawasaki et al., 1988). The primers used at baseline were: sense: 5' GGAGCTGCAGATGCTGACAAC 3' and antisense: 5' TCAGACCCTGAGGCTCAAAGTC 3 ' (Kawasaki et al., 1988). Nested RT-PCR was used in follow-up cases for monitoring for minimal residual disease post-imatinib (Gleevac) treatment at intervals of 3, 6, 12, 18, and 24 months (Cross et al., 1993). The primers used for first round nested RT-PCR were: NB1: 5' GAGCGTGCAGAGTGGAGGGAGAACA 3', ABL3: 5' GGTACCAGGAGTGTTTCTCCAGACTG 3'. The primers used for second round nested RT-PCR were B2A: 5' TTCAGAAGCTTCTCCCTGACAT 3', CA3: 5' TGTTGACTGGCGTGATGTAGTTGCTTGG 3'.

Since the two groups, i.e., $b 3 a 2$ and $b 2 a 2$, were not equal in number, we used the SPSS software version 14, multinomial regression logistic analysis to study the likelihood odds ratios (OR) between the two transcripts across different variable groups and calculated the statistical significance. All these cases were also analyzed at different follow-up intervals by RT-PCR and FISH, to determine the prognostic significance of both isoforms.

\section{RESULTS}

\section{Incidence of $b 3 a 2$ and $b 2 a 2$}

Of the 202 CML patients, $138(68 \%)$ had the $b 3 a 2$ type bcr/abl transcript and 64 (32\%) had the $b 2 a 2$ type, with the ratio being 2:1. After RT-PCR, types $b 3 a 2$ and $b 2 a 2$ gave rise to amplified DNA fragments of 200 and $125 \mathrm{bp}$, respectively, using the above-mentioned primers at baseline, and 460 and $385 \mathrm{bp}$, respectively, for nested RT-PCR. The male to female 
ratio was 3:1 in $b 3 a 2$ and 2:1 in $b 2 a 2$, and the incidence of both isoforms was higher in adults than in children (132/138 in $b 3 a 2$ and 61/64 in b2a2).

\section{Clinical findings}

The clinical parameter hepatosplenomegaly was studied in 106 patients, of whom 70 had $b 3 a 2$ and 36 had $b 2 a 2$ (ratio being 2:1). There was not much difference between the two transcripts with regard to size of spleen or liver.

\section{Hematological findings}

We analyzed the transcripts in different ranges of hemoglobin and leukocyte and platelet counts to find out whether there was any significant difference in the hematological findings between the transcript types.

The relationship between the two transcripts and the patients' hematological findings at diagnosis were studied in 106 patients, of whom 70 had $b 3 a 2$ and 36 had b2a2 (ratio being 2:1). There was no correlation between the hemoglobin distribution and the type of transcripts. The median range for hemoglobin concentration was between 5 and $10 \mathrm{~g} / \mathrm{dL}$. TLC ranging from less than $20 \times 10^{9} / \mathrm{L}$ to more than $400 \times 10^{9} / \mathrm{L}$ was observed with a median range of 100 to $200 \times 10^{9} / \mathrm{L}$ in the two transcripts. We analyzed the distribution of both transcripts in each of the following ranges as shown in Table 1, and calculated the OR to find out whether there was any significant difference. We did not find any difference between the two transcripts in terms of TLC.

\begin{tabular}{|c|c|c|}
\hline & $b 3 a 2(\mathrm{~N}=70)$ & $b 2 a 2(\mathrm{~N}=36)$ \\
\hline \multicolumn{3}{|l|}{ TLC } \\
\hline Median range & 100 to $200 \times 10^{9} / \mathrm{L}$ & 100 to $200 \times 10^{9} / \mathrm{L}$ \\
\hline Less than $50 \times 10^{9} / \mathrm{L}$ & $23(33 \%), \mathrm{OR}=1.06$ & $11(31 \%), \mathrm{OR}=0.94$ \\
\hline 50 to $100 \times 10^{9} / \mathrm{L}$ & $7(10 \%), \mathrm{OR}=0.71$ & $5(14 \%), \mathrm{OR}=1.40$ \\
\hline More than $100 \times 10^{9} / \mathrm{L}$ & $40(57 \%), \mathrm{OR}=1.03$ & $20(55 \%), \mathrm{OR}=0.97$ \\
\hline \multicolumn{3}{|l|}{ Platelets } \\
\hline Median range & 300 to $400 \times 10^{9} / \mathrm{L}$ & 300 to $400 \times 10^{9} / \mathrm{L}$ \\
\hline Less than $100 \times 10^{9} / \mathrm{L}$ & $4(6 \%), \mathrm{OR}=0.75$ & $3(8 \%), \mathrm{OR}=1.33$ \\
\hline 100 to $400 \times 10^{9} / \mathrm{L}$ & $32(45.5 \%), \mathrm{OR}=0.81$ & $20(56 \%), \mathrm{OR}=1.23$ \\
\hline 400 to $1000 \times 10^{9} / \mathrm{L}$ & $28(40 \%), \mathrm{OR}=1.33$ & $11(30 \%), \mathrm{OR}=0.75$ \\
\hline More than $1000 \times 10^{9} / \mathrm{L}$ & $6(8.5 \%), \mathrm{OR}=1.4 \mathrm{l}$ & $2(6 \%), \mathrm{OR}=0.70$ \\
\hline
\end{tabular}

OR $=$ odds ratio.

Platelet count ranging from less than $100 \times 10^{9} / \mathrm{L}$ to more than $1000 \times 10^{9} / \mathrm{L}$ was observed with a median range of 300 to $400 \times 10^{9} / \mathrm{L}$ in the two transcripts. Again, we did not find any significant difference between the two transcripts. Even after stratifying on the basis of platelet count of more than $1000 \times 10^{9} / \mathrm{L}$, we did not find any significant association between the transcripts and thrombocythemia, as shown in Table 1.

We further stratified the patients on the basis of platelet count above or below $400 \mathrm{x}$ $10^{9} / \mathrm{L}$ and TLC above or below $100 \times 10^{9} / \mathrm{L}$, in both transcripts. We found that in both transcripts, increased platelet count of more than $400 \times 10^{9} / \mathrm{L}$ was significantly associated with high TLC of more than $100 \times 10^{9} / \mathrm{L}$ (for $b 3 a 2, \mathrm{OR}=2$ and for $b 2 a 2, \mathrm{OR}=2.5$ ) as shown in Table 2 . 
Table 2. Correlation between total leukocyte count (TLC), platelet count and transcripts.

\begin{tabular}{|c|c|c|c|c|}
\hline \multirow[t]{3}{*}{ Platelet count } & \multicolumn{4}{|c|}{ TLC } \\
\hline & \multicolumn{2}{|c|}{$b 3 a 2(\mathrm{~N}=70)$} & \multicolumn{2}{|c|}{$b 2 a 2(\mathrm{~N}=36)$} \\
\hline & $<100 \times 10^{9} / \mathrm{L}$ & $>100 \times 10^{9} / \mathrm{L}$ & $<100 \times 10^{9} / \mathrm{L}$ & $>100 \times 10^{\circ} / \mathrm{L}$ \\
\hline$<400 \times 10^{\circ} / \mathrm{L}(\mathrm{N}=59)$ & $19(28 \%), \mathrm{OR}=0.80$ & $17(35 \%), \mathrm{OR}=1.25$ & $12(33 \%), \mathrm{OR}=1.06$ & $11(31 \%), \mathrm{OR}=0.94$ \\
\hline$>400 \times 10^{\circ} \mathrm{L}(\mathrm{N}=46)$ & $11(16 \%), \mathrm{OR}=0.50$ & $23(21 \%), O R=2$ & $4(11 \%), O R=0.40$ & $9(25 \%), \mathrm{OR}=2.50$ \\
\hline
\end{tabular}

\section{Correlation of transcripts and bone marrow morphology}

Bone marrow morphology for blast, basophil and eosinophil percentages, and phase of CML disease, chronic phase, accelerated phase and blast crisis were studied in 173 patients, of whom 118 had $b 3 a 2$ and 55 had $b 2 a 2$ (ratio being 2:1). It was seen that eosinophilia and basophilia (more than 5\%) were marginally associated with $b 3 a 2$, but the findings were not significant. However, there was no correlation between the bone marrow blast percentage and the two transcripts. It was also seen that $11 \%$ of $b 2 a 2(\mathrm{OR}=2.2)$ presented CML blast crisis as compared to $5 \%$ of $b 3 a 2(\mathrm{OR}=0.4)$. This was a significant finding (Table 3$)$. Among the blast crisis group, 5 of 7 patients with myeloid blast crisis had $b 2 a 2$, while 3 of 4 patients with lymphoid blast crisis had $b 3 a 2$, which was again significant.

Table 3. Correlation between phase of chronic myelogenous leukemia (CML) and transcripts.

\begin{tabular}{lcccc}
\hline & \multicolumn{4}{c}{ Phase of CML disease } \\
\cline { 2 - 5 } & CML CP $(\mathrm{N}=148)$ & CML AP $(\mathrm{N}=14)$ & CML myeloid BC $(\mathrm{N}=7)$ & CML lymphoid BC (N = 4) \\
\hline$b 3 a 2(\mathrm{~N}=118)$ & $103(87 \%)$ & $10(8 \%)$ & $2(2 \%)$ & $3(3 \%)$ \\
$b 2 a 2(\mathrm{~N}=55)$ & $45(82 \%)$ & $4(7 \%)$ & $5(9 \%)$ & $1(2 \%)$ \\
\hline
\end{tabular}

$\overline{\mathrm{CP}}=$ chronic phase; $\mathrm{AP}=$ accelerated phase; $\mathrm{BC}=$ blast crisis.

\section{Correlation of transcripts and FISH}

The two transcripts were correlated with FISH in 186 patients (of whom 125 had $b 3 a 2$ and 61 had $b 2 a 2$, ratio being 2:1), to find out whether any of the additional chromosomal abnormalities such as 9q deletion, $5^{\prime} \mathrm{Abl}$ deletion or Ph duplication had implications on any of the transcripts. It was observed that $10 \%$ of $b 3 a 2$ had $5^{\prime} \mathrm{Abl}$ deletion $(\mathrm{OR}=3.3)$ as compared to $3 \%$ of $b 2 a 2(\mathrm{OR}=0.3)$, as shown in Table 4 . This was a significant observation.

Table 4. Correlation between fluorescent in situ hybridization (FISH) results and transcripts.

\begin{tabular}{lccccc}
\hline & \multicolumn{5}{c}{ FISH } \\
\cline { 2 - 6 } & $\mathrm{t}(9,22)(\mathrm{N}=136)$ & $9 \mathrm{q}$ deletion $(\mathrm{N}=23)$ & Variant $\mathrm{Ph}(\mathrm{N}=10)$ & $5^{\prime}$ abl deletion $(\mathrm{N}=15)$ & Ph duplication $(\mathrm{N}=2)$ \\
\hline$b 3 a 2(\mathrm{~N}=125)$ & $89(70.5 \%)$ & $14(11 \%)$ & $7(5.5 \%)$ & $13(10 \%)$ & $2(3 \%)$ \\
$b 2 a 2(\mathrm{~N}=61)$ & $47(77 \%)$ & $9(15 \%)$ & $3(5 \%)$ & $2(3 \%)$ & 0 \\
\hline
\end{tabular}




\section{Post-Gleevac thrombocytopenia}

Post-Gleevac thrombocytopenia of less than $100 \times 10^{9} / \mathrm{L}$ was studied in 97 patients, of whom 63 had $b 3 a 2$ and 34 had $b 2 a 2$ (ratio being 1.85:1). It was associated more with $b 2 a 2(44 \%, \mathrm{OR}=1.76)$ than with $b 3 a 2(25 \%, \mathrm{OR}=0.56)$. We further examined whether the initial platelet count at baseline had any role in the development of thrombocytopenia, after starting of treatment with Gleevac, or if it was because of the transcript type. We also tried to find out the association of initial platelet count with the time at which thrombocytopenia developed. The initial platelet count at the time of presentation did have a significant impact on the development of thrombocytopenia, as shown in Table 5. The higher the platelet count, the lower the likelihood was of developing thrombocytopenia, and even if it developed, it occurred with longer duration of treatment. Upon stratifying on the basis of transcript type and platelet count above or below $400 \times 10^{9} / \mathrm{L}$, the difference was again more because of platelet count, while the percentage of the two transcripts developing thrombocytopenia was almost equal in both groups, as shown in Table 6. Thus, it could be concluded that post-Gleevac thrombocytopenia was because of the initial platelet count at baseline, and was not significantly associated with transcript type.

Table 5. Correlation between post-Gleevac thrombocytopenia and platelet count.

\begin{tabular}{|c|c|c|c|c|c|}
\hline \multirow[t]{2}{*}{ Platelets $(\mathrm{N}=97)$} & \multicolumn{5}{|c|}{ Post-Gleevac thrombocytopenia } \\
\hline & No & less than 3 months & 3 to 6 months & 6 months to 1 year & more than 1 year \\
\hline less than $<100 \times 10^{9} / \mathrm{L}(\mathrm{N}=4)$ & $1(25 \%)$ & $3(75 \%)$ & 0 & 0 & 0 \\
\hline 100 to $200 \times 10^{9} / \mathrm{L}(\mathrm{N}=14)$ & $6(43 \%)$ & $2(14 \%)$ & $2(14 \%)$ & 0 & $4(29 \%)$ \\
\hline 200 to $300 \times 10^{9} / \mathrm{L}(\mathrm{N}=24)$ & $16(67 \%)$ & $5(21 \%)$ & 0 & 0 & $3(12 \%)$ \\
\hline 300 to $400 \times 10^{9} / \mathrm{L}(\mathrm{N}=10)$ & $7(70 \%)$ & 0 & 0 & 0 & $3(30 \%)$ \\
\hline 400 to $100 \times 10^{9} / \mathrm{L}(\mathrm{N}=38)$ & $29(76 \%)$ & $3(8 \%)$ & 1 & $2(5 \%)$ & $3(8 \%)$ \\
\hline More than $100 \times 10^{\circ} \mathrm{L}(\mathrm{N}=7)$ & $7(100 \%)$ & 0 & 0 & 0 & 0 \\
\hline
\end{tabular}

Table 6. Correlation between thrombocytopenia and transcripts.
\begin{tabular}{lcc}
\hline Platelets & $b 3 a 2(\mathrm{~N}=16)$ & $b 2 a 2(\mathrm{~N}=15)$ \\
\hline$<400 \times 10^{\circ} \mathrm{L}$ & $12(75 \%)$ & $12(80 \%)$ \\
$>400 \times 10^{\circ} / \mathrm{L}$ & $4(25 \%)$ & $3(20 \%)$ \\
\hline
\end{tabular}

\section{Response to treatment}

We analyzed 70 patients for molecular response at 6 months and 67 patients at 1 year. Though $48 \%$ of patients with $b 3 a 2$ were in molecular remission at 6 months as compared to $41 \%$ patients with $b 2 a 2$, the difference was not significant (Table 7). On the other hand, $76 \%$ of $b 2 a 2$ showed molecular remission at 1 year as compared to $55 \%$ of $b 3 a 2$, but again this was not significant (Table 8). 


\begin{tabular}{lcc} 
Table 7. Molecular response at 6 months as analyzed by RT-PCR. & \\
\hline & Positive & Negative \\
\hline$b 3 a 2(\mathrm{~N}=48)$ & $25(52 \%)$ & $23(48 \%)$ \\
$b 2 a 2(\mathrm{~N}=22)$ & $\mathrm{OR}=0.88$ & $\mathrm{OR}=1.17$ \\
& $13(59 \%)$ & $9(41 \%)$ \\
& $\mathrm{OR}=1.13$ & $\mathrm{OR}=0.85$ \\
\hline
\end{tabular}

Table 8. Molecular response at 1 year as analyzed by RT-PCR.

\begin{tabular}{|c|c|c|}
\hline & Positive & Negative \\
\hline \multirow[t]{2}{*}{$b 3 a 2(\mathrm{~N}=55)$} & $25(45 \%)$ & $30(55 \%)$ \\
\hline & $\mathrm{OR}=1.87$ & $\mathrm{OR}=0.72$ \\
\hline \multirow[t]{2}{*}{$b 2 a 2(\mathrm{~N}=12)$} & $3(24 \%)$ & $9(76 \%)$ \\
\hline & $\mathrm{OR}=0.53$ & $\mathrm{OR}=1.38$ \\
\hline
\end{tabular}

Cytogenetic response was also studied in 96 patients at 6 months, and in 85 patients at 1 year from initiation of treatment with Gleevac. The response was classified as complete, major, minor, or no response as described elsewhere in the literature (Druker et al., 2003). Again, there was not much significant difference in the cytogenetic response between the two transcripts, at 6 months and 1 year (data not shown). We also correlated molecular response with initial TLC and platelet count, but there was no significant association, neither in the whole population nor within the transcript groups (data not shown).

\section{DISCUSSION}

There have been many contrasting studies reported in literature, correlating type of $b c r / a b l$ mRNA and hematological parameters in patients with CML. The differences seen in these studies may be explained by differences in sample size, sample selection and bias or due to racial differences. All studies published so far have used the Student $t$-test for analysis. They calculated the mean and the median in each group across different variables studied and calculated the $P$ value. However, there was no mention in these studies of the actual percentage of patients in each transcript group, which showed differences in the variables or parameters studied, and whether this was significant or not. In all these studies, the number of patients with $b 3 a 2$ was more than $b 2 a 2$, and this could have led to bias towards $b 3 a 2$. We had the opportunity to study a large number of patients at our institution who were treated with imatinib myselate, also known as Gleevac. To overcome the selection bias towards any of the groups, we applied multinomial regression logistic analysis to determine the likelihood OR between the two transcripts across different variables or parameters studied, and calculated the statistical significance using the SPPS software, version 14. We divided the different hematological parameters hemoglobin, TLC and platelet count into different ranges and studied the percentage of patients in the two transcripts across each range of hematological parameter and then calculated the OR and the $P$ value to establish any significant association.

In accordance with other studies in the literature (Martiat et al., 1989; Inokuchi et al., 1991a,b; Opalka et al., 1992; Shepherd et al., 1992; Rozman et al., 1995; Perego et al., 2000; de 
Lemos et al., 2005), there was no correlation between the two transcripts and liver and spleen size and hemoglobin level. We tried to analyze the relationship between TLC, platelet count and the two transcripts using the OR. Although $b 3 a 2$ did show a higher association with high TLC ranging from 100 to $400 \times 10^{9} / \mathrm{L}$, compared to $b 2 a 2$, it was not significant (OR for $b 3 a 2$ was 1.5 and 0.7 , for $b 2 a 2$ ). This finding was similar to that reported in the literature (Martiat et al., 1989; Inokuchi et al., 1991a,b; Opalka et al., 1992; Shepherd et al., 1992; Rozman et al., 1995; Perego et al., 2000; de Lemos et al., 2005). In analyzing the relationship between platelet count and the two transcripts, again we did find a slightly better association of $b 3 a 2$ with high platelet count of more than $400 \times 10^{9} / \mathrm{L}$, than for $b 2 a 2$, but again it was not significant (OR for $b 3 a 2$ was 1.3 and 0.7 for $b 2 a 2$ ). Some studies have shown that patients with the $b 3 a 2$ transcript have a higher platelet count than patients with the $b 2 a 2$ transcript (Martiat et al., 1989; Inokuchi et al., 1991a,b; Perego et al., 2000; de Lemos et al., 2005). However, others were not able to establish any relationship between the two (Shepherd et al., 1992; Rozman et al., 1995).

One of the reasons for these contrasting results could be the sample size. Many of the studies, which showed a significant association between platelet count and $b 3 a 2$, had a small patient population ranging from less than 50 to 80 . Because of this small number, there could have been a bias towards $b 3 a 2$ since the incidence of $b 3 a 2$ is higher than $b 2 a 2$. However, in our study, we analyzed 106 patients, which is a good sample number for determining significant results. Many studies have shown that there is an association between $b 3 a 2$ with high platelet count of more than $1000 \times 10^{9} / \mathrm{L}$ in patients with TLC less than $100 \times 10^{9} / \mathrm{L}$ (Martiat et al., 1989; Shepherd et al., 1992; Perego et al., 2000; de Lemos et al., 2005), but again, the number of patients in these studies was very small ( 5 of 6 patients in the study conducted by Martiat et al., 1989, and 3 patients in another study conducted by Shepherd et al., 1992). However, in contrast to what has been reported in these studies, we found that high platelet count was associated with high TLC irrespective of transcript type. This could be explained by the postulation that the inclusion of the $\mathrm{Dbl} / \mathrm{PH}$ domains in the bcr region in $p 210$ and $p 230$ may contribute to the granulocytic differentiation associated with these variants (Advani and Pendergast, 2002), resulting in hyperleukocytosis and thrombocytosis.

To resolve the contrasting data suggesting a possible relationship between $\mathrm{Bcr} / \mathrm{Abl}$ protein structure and thrombopoiesis, further studies are required. Although one study did establish a possible correlation between $b 3 a 2$ and thrombopoiesis by studying the interaction between the actin and microtubule systems and megakaryocyte integrins and the extracellular matrix, and found that rare patients with Ph-positive essential thrombocythemia with the highest platelet counts correlated with the $b 3 a 2$ type transcript (Perego et al., 2000). However, it was noteworthy that even in this study, there was a subgroup among the $b 3 a 2$ patients with platelet counts not different from those of the $b 2 a 2$ group. Thus, in the multifactorial process of platelet production, there could be many signaling pathways that interact with each other at different levels, and the type of $B c r / A b l$ rearranged product alone is not sufficient to explain the increase in thrombopoiesis. It is possible that the signal transduction role of $B c r / A b l$ protein, where present, would only be a variable in certain cases, influencing the phenomenon. An example of this multiple involvement of different pathways in thrombopoiesis is provided by essential thrombocythemia, a clonal disorder characterized by thrombocytosis and abnormal proliferation of megakaryocytes, and in which the $\mathrm{Bcr} / \mathrm{Abl}$ protein is not present in the great majority of cases. In this disease, it seems that an altered expression of thrombopoietin and its receptor c-Mpl or an altered sensitivity of the thrombopoietin-c-Mpl system may play a role (Matsumura et al., 1999), although their exact involvement in thrombopoiesis is still elusive. 
We correlated bone marrow aspirate findings of blast, basophil and eosinophil percentages and the phase of the disease, with the two transcripts to find out whether the 5' or 3' breakpoints in the bcr region had any influence on myeloid cell proliferation. Though there have been reports correlating the phase of CML disease with the transcripts (Shtalrid et al., 1988; Mills et al., 1988, 1991; Shepherd et al., 1995; Elliott et al., 1995; Melo, 1996a), none of the studies have correlated bone marrow findings with the two transcripts. We did not find any significant difference between the two fusion transcripts when correlating them with the percentage of myeloid cells in the marrow, indicating that there is no difference in the role of the two fusion transcripts in myeloid cell proliferation. In correlating with phase of the disease, we found that there was not much difference between the two transcripts among the patients who were in chronic phase. However, we found that $b 2 a 2$ was significantly associated with blast crisis $(\mathrm{OR}=2.2)$ compared to $b 3 a 2(\mathrm{OR}=0.4)$. This is interesting, given that the longer $B c r / A b l$ variants (i.e., p230) tend to have a more indolent biological/clinical phenotype than the shorter variants (i.e., p185). Even among the group of patients who presented blast crisis, $b 2 a 2$ was significantly associated with myeloid blast crisis (5 of 7), and $b 3 a 2$ was associated with lymphoid blast crisis ( 3 of 4$)$. There are some data indicating that patients with $b 3 a 2$ exhibit a long chronic phase compared to those with $b 2 a 2$ (Mills et al., 1988; Shepherd et al., 1995; Elliott et al., 1995; Melo, 1996a), while others have described that patients with $b 3 a 2$ exhibit a short chronic phase (Shtalrid et al., 1988; Mills et al., 1991). One of these studies analyzed the genomic break points in the bcr region (F0 to F4) and found that there was a trend for longer duration of chronic phase in patients with breakpoints in the F2 region as compared to other regions. However, there was no statistical difference in the duration of the chronic phase in patients with breakpoints in the F1 and F2 regions (corresponding to $b 2 a 2 \mathrm{mRNA}$ ) and with the $\mathrm{F} 3$ and $\mathrm{F} 4$ regions (corresponding to b3a2 mRNA) (Mills et al., 1991). The same study also showed that there was no difference in the number of patients with blast crisis for both regions, i.e., F1 and F2 or F3 and F4. However, patients with F1 and F2 breakpoint regions (b2a2) had slightly higher association with myeloid blast crisis, while patients with F3 and F4 breakpoint regions (b3a2) had an association with lymphoid blast crisis. This finding was similar to that in our study.

We also examined correlations with FISH findings to find out whether either transcript showed any significant cytogenetic abnormalities. Interestingly, 5' Abl deletion was significantly associated with $b 3 a 2(\mathrm{OR}=3.3)$ compared to $b 2 a 2(\mathrm{OR}=0.3)$. No previous reports have analyzed this association. The incidence of $5^{\prime} \mathrm{Abl}$ deletion is around $10 \%$, as reported in literature (Huntly et al., 2003). However, the association of $b 3 a 2$ with 5' $A b l$ deletion needs further investigations. There is one study where it was reported that 3 of 4 patients had a breakpoint 5 , of $A b l$ gene and had a $b 3 a 2$ type junction. In these patients, the entire $A b l$ gene was translocated to chromosome 22, and these patients had essential thrombocytosis (Morris et al., 1991).

After initiation of treatment with imatinib myselate, we again analyzed the TLC and platelet count to see whether there was any difference in the response of patients between these two transcripts. One interesting observation was that post-Gleevac thrombocytopenia (less than $100 \times 10^{9} / \mathrm{L}$ ), which is a common hematological finding during treatment with imatinib, was seen more with $b 2 a 2(\mathrm{OR}=1.76)$ than with $b 3 a 3(\mathrm{OR}=0.5)$. However, on correlating with the initial platelet count at baseline, we found that the association of thrombocytopenia was higher with low platelet counts at baseline than with the transcripts. It was seen that, the higher the platelet count at baseline, the lower the chances are of developing thrombocytope- 
nia. Interestingly, all patients with platelet counts of more than $1000 \times 10^{9} / \mathrm{L}$ did not show postGleevac thrombocytopenia at all. This finding has not been previously reported in literature.

On analyzing the therapeutic response based on molecular and cytogenetic response, we did not find any difference between the two transcripts at 6 months or 1 year. There are some data indicating that patients with $b 3 a 2$ have a better prognosis than with $b 2 a 2$, exhibiting a long chronic phase and better response to interferon (Mills et al., 1988, 1991; Elliott et al., 1995; Melo, 1996a). On the other hand, a recent study showed that patients with $b 2 a 2$ had a better molecular response than with $b 3 a 2$ at 6 months and at 1 year (de Lemos et al., 2005). However, other prospective studies have suggested no difference in prognosis between patients with $b 2 a 2$ versus b3a2 transcripts (Fioretos et al., 1993; Rozman et al., 1995; Shepherd et al., 1995). High platelet counts greater than $1000 \times 10^{9} / \mathrm{L}$ at diagnosis have been recognized as a poor prognostic marker and an early marker of accelerated phase (Shtalrid et al., 1988; Morris et al., 1990). However, we did not find any such association, and patients even with a platelet count of more than $1000 \times 10^{9} / \mathrm{L}$ achieved complete cytogenetic remission at the end of 6 months ( 4 of 6 , $67 \%$ ) and at one year $(3$ of 5, 60\%). All these initial reports published in the literature were before the use of imatinib, wherein patients were treated with interferon and/or hydroxyurea and found a better response in patients with $b 3 a 2$ than with $b 2 a 2$ and in patients with low platelet counts. However, now, perhaps due to the advent of imatinib, the type of transcript or the initial platelet count does not have any implications on the overall molecular remission. This could be explained by a study in which a phase 1 trial was carried out with 54 patients with CML refractory to interferon- $\alpha$. Of these patients, $23 / 24$ patients receiving a dose of $300 \mathrm{mg}$ or higher of imatinib underwent a complete hematological remission, and 8/24 had a complete cytogenetic remission (Druker et al., 1999). Imatinib is a signal transduction inhibitor (STI-571), with potent tyrosine kinase inhibition and selectivity for $\mathrm{Abl}, \mathrm{c}-\mathrm{Kit}$, and platelet-derived growth factor. STI-571 binds to a pocket of the catalytic domain of the Abl tyrosine kinase and competitively inhibits binding of adenosine triphosphate, thereby resulting in inhibition of autophosphorylation and inhibition of substrate phosphorylation (Buchdunger et al., 1996; Schindler et al., 2000). Since imatinib does not have any role in the inhibition of the bcr region, the type of breakpoint in the bcr region may not influence the response to treatment with imatinib.

In conclusion, our findings confirm that $b 3 a 2$ type transcript is not significantly associated with thrombopoiesis, and that there is no difference in treatment response between the two transcripts. However, further studies are required to understand the molecular pathways involved in the $\mathrm{Bcr} / \mathrm{Abl}$ mechanism. In addition, the significant findings from our study were the association of blast crisis with $b 2 a 2$, especially with myeloid blast crisis, association of 5 , $A b l$ deletion with $b 3 a 2$ and association of post-imatinib thrombocytopenia with low platelet count at baseline. These findings have not been previously reported in the literature and therefore need further studies and validation.

\section{REFERENCES}

Acar H, Stewart J and Connor MJ (1997). Philadelphia chromosome in chronic myelogenous leukemia: confirmation of cytogenetic diagnosis in $\mathrm{Ph}$ positive and negative cases by fluorescence in situ hybridization. Cancer Genet. Cytogenet. 94: 75-78.

Advani AS and Pendergast AM (2002). Bcr-Abl variants: biological and clinical aspects. Leuk. Res. 26: 713-720.

Buchdunger E, Zimmermann J, Mett H, Meyer T, et al. (1996). Inhibition of the Abl protein-tyrosine kinase in vitro and in vivo by a 2-phenylaminopyrimidine derivative. Cancer Res. 56: 100-104. 
Cross NC, Hughes TP, Feng L, O'Shea P, et al. (1993). Minimal residual disease after allogeneic bone marrow transplantation for chronic myeloid leukaemia in first chronic phase: correlations with acute graft-versus-host disease and relapse. Br. J. Haematol. 84: 67-74.

de Lemos JA, de Oliveira CM, Scerni AC, Bentes AQ, et al. (2005). Differential molecular response of the transcripts B2A2 and B3A2 to imatinib mesylate in chronic myeloid leukemia. Genet. Mol. Res. 4: 803-811.

Druker BJ, Peng B, Resta D, Sawyers CL, et al. (1999). Phase I trial of a specific ABL tyrosine kinase inhibitor, CGP 57148, in interferon refractory. Proc. Am. Soc. Clin. Oncol. 18: 7a.

Druker BJ, Gathmann I, Bolton AE and Larson RA (2003). Probability and impact of obtaining a cytogenetic response to imatinib as initial therapy for chronic myeloid leukemia (CML) in chronic phase. Blood 102: 182.

Elliott SL, Taylor KM, Taylor DL, Rodwell RL, et al. (1995). Cytogenetic response to alpha-interferon is predicted in early chronic phase chronic myeloid leukemia by M-bcr breakpoint location. Leukemia 9: 946-950.

Fioretos T, Nilsson PG, Aman P, Heim S, et al. (1993). Clinical impact of breakpoint position within M-bcr in chronic myeloid leukemia. Leukemia 7: 1225-1231.

Huntly BJ, Bench A and Green AR (2003). Double jeopardy from a single translocation: deletions of the derivative chromosome 9 in chronic myeloid leukemia. Blood 102: 1160-1168.

Inokuchi K, Inoue T, Tojo A, Futaki M, et al. (1991a). A possible correlation between the type of bcr-abl hybrid messenger RNA and platelet count in Philadelphia-positive chronic myelogenous leukemia. Blood 78: 3125-3127.

Inokuchi K, Futaki M, Yamada T, Tanabe Y, et al. (1991b). The relationship between the site of breakpoints within the bcr gene and thrombopoiesis of Philadelphia-positive chronic myelocytic leukemia. Leuk. Res. 15: 1067-1073.

Kawasaki ES, Clark SS, Coyne MY, Smith SD, et al. (1988). Diagnosis of chronic myeloid and acute lymphocytic leukemias by detection of leukemia-specific mRNA sequences amplified in vitro. Proc. Natl. Acad. Sci. U. S. A. 85: 5698-5702.

Kratz A, Ferraro M, Sluss PM and Lewandrowski KB (2004). Case records of the Massachusetts General Hospital. Weekly clinicopathological exercises. Laboratory reference values. N. Engl. J. Med. 351: 1548-1563.

Kurzrock R, Gutterman JU and Talpaz M (1988). The molecular genetics of Philadelphia chromosome-positive leukemias. N. Engl. J. Med. 319: 990-998.

Lewis SM (2001). References and Normal Values. In: Dacie and Lewis Practical Hematology (Lewis SM, Bain BJ and Bates I, eds.). Churchill Livingstone, Philadelphia, 9-18.

Martiat P, Ifrah N, Rassool F, Morgan G, et al. (1989). Molecular analysis of Philadelphia positive essential thrombocythemia. Leukemia 3: 563-565.

Matsumura I, Horikawa Y and Kanakura Y (1999). Functional roles of thrombopoietin-c-mpl system in essential thrombocythemia. Leuk. Lymphoma 32: 351-358.

Melo JV (1996a). The diversity of BCR-ABL fusion proteins and their relationship to leukemia phenotype. Blood 88: 2375-2384.

Melo JV (1996b). The molecular biology of chronic myeloid leukaemia. Leukemia 10: 751-756.

Mills KI, MacKenzie ED and Birnie GD (1988). The site of the breakpoint within the bcr is a prognostic factor in Philadelphia-positive CML patients. Blood 72: 1237-1241.

Mills KI, Sproul AM, Leibowitz D and Burnett AK (1991). Mapping of breakpoints, and relationship to BCR-ABL RNA expression, in Philadelphia-chromosome-positive chronic myeloid leukaemia patients with a breakpoint around exon 14 (b3) of the BCR gene. Leukemia 5: 937-941.

Morris SW, Daniel L, Ahmed CM, Elias A, et al. (1990). Relationship of ber breakpoint to chronic phase duration, survival, and blast crisis lineage in chronic myelogenous leukemia patients presenting in early chronic phase. Blood 75: 2035-2041.

Morris CM, Heisterkamp N, Groffen J and Fitzgerald PH (1991). Entire ABL gene is joined with 5'-BCR in some patients with Philadelphia-positive leukemia. Blood 78: 1078-1084.

Nowell PC and Hungerford DA (1960). A minute chromosome in human chronic granulocytic leukemia. Science 132: 1497-1501.

Opalka B, Wandl UB, Stutenkemper R, Kloke O, et al. (1992). No correlation between the type of bcr-abl hybrid messenger RNA and platelet counts in chronic myelogenous leukemia. Blood 80: 1854-1855.

Perego RA, Costantini M, Cornacchini G, Gargantini L, et al. (2000). The possible influences of B2A2 and B3A2 BCR/ ABL protein structure on thrombopoiesis in chronic myeloid leukaemia. Eur. J. Cancer 36: 1395-1401.

Rowley JD (1973). Letter: A new consistent chromosomal abnormality in chronic myelogenous leukaemia identified by quinacrine fluorescence and Giemsa staining. Nature 243: 290-293.

Rozman C, Urbano-Ispizua A, Cervantes F, Rozman M, et al. (1995). Analysis of the clinical relevance of the breakpoint location within M-BCR and the type of chimeric mRNA in chronic myelogenous leukemia. Leukemia 9: 1104-1107.

Schindler T, Bornmann W, Pellicena P, Miller WT, et al. (2000). Structural mechanism for STI-571 inhibition of abelson tyrosine kinase. Science 289: 1938-1942. 
Shepherd PC, Bond C and Allan NC (1992). Molecular breakpoints and platelet counts in chronic myeloid leukemia. Blood 80: 556-557.

Shepherd P, Suffolk R, Halsey J and Allan N (1995). Analysis of molecular breakpoint and m-RNA transcripts in a prospective randomized trial of interferon in chronic myeloid leukaemia: no correlation with clinical features, cytogenetic response, duration of chronic phase, or survival. Br. J. Haematol. 89: 546-554.

Shtalrid M, Talpaz M, Kurzrock R, Kantarjian H, et al. (1988). Analysis of breakpoints within the bcr gene and their correlation with the clinical course of Philadelphia-positive chronic myelogenous leukemia. Blood 72: 485-490.

Specchia G, Mininni D, Guerrasio A, Palumbo G, et al. (1995). Ph positive acute lymphoblastic leukemia in adults: molecular and clinical studies. Leuk. Lymphoma 18 (Suppl. 1): 37-42. 